replaced by an ointment which is rubbed into the patch twice a day, and allowed to remain on. The formula for the ointment is as follows: Caustic potash gr. ix., carbolic acid gr. xxiv., lanoline and oil of cocoanut of each zss., to be well rubbed together. S.-A small portion to be rubbed in night and morning. The hair, as in the former plan, is cut short, but not shaved, and the treatment is persistently carried out for a period of from one to three months. Tne healthy parts of the scalp may be dressed with a pomade containing boric acid ointment and eucalyptus ointment, of each two parts in six. This may be used as a preventative on the heads of the persons with whom the child lives. "There is generally improvement after two or three weeks treatment, the conidia are fewer in number, and the bare patches begin to show new hairs. The accompanying photographs are taken from cases at different stages of treatment by the ointment. The first shows the state when the patient was first seen, the hair being erowded with spores.. From the same patient, but a month later, the second one was taken. The diminution in the numbers of the parasites is very marked, but the structure of the hair is partly destroyed in the usual manner. The third shows a damaged hair from

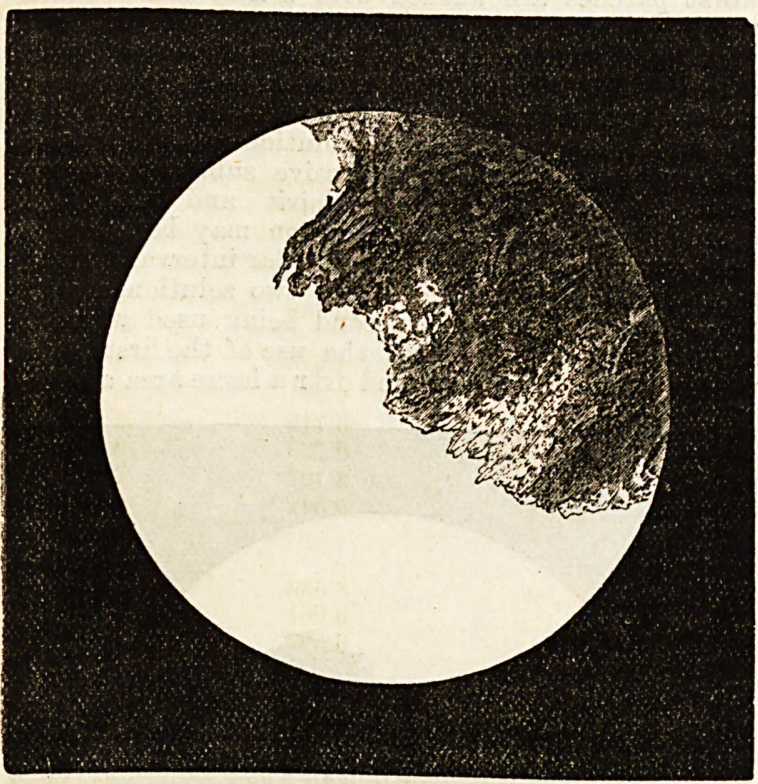

FIG. 3.-End of a hair, frayed out, after treatment. (A. J. Harrison.)

another case where the disease had almost entirely disappeared after six weeks dressing, and a complete cure followed. We are indebted to Dr. Harrison for allowing us to reproduce these engravings from a former paper read before the British Medical Association in 1888, and published in the journal for 1889 . Both methods have been in constant use since then with most satisfactory results. Under any remedy much care is required to ensure constant regular dressing of every patch. Finally, hairs should be tested after the treatment has been carried on some time to discover the presence of spores. This may be done under the microscope, or by cultivation, or even by their turning white, if diseased, when dipped in chloroform. The advantage of cultivation on a faintly acid solution of dextrose is that a score of hairs may be dropped on to the medium together, and a growth existing in any one of them will be detected without more trouble.

Whatever treatment we adopt it must be continued until no trace of the disease remains, and until no recurrence is seen after many weeks' rest. Meanwhile children may be even allowed at school if close caps are worn, and the hair cut short, and dressed with strong boric solution or ointment, and washed with soft soap frequently. Practically, however, caps are thrown off in play, and dresses become infected so that isolation is on the whole safer.

It will be seen that all these plans of treatment aim at a general disinfection of the surface, combined with powerful remedies directed to the worst patches. These may be simple antiseptics, or may act by depriving the the plant of the water and oxygen necessary for its life. Possibly some antiseptic like alumnol, which is not precipitated by an excess of albumen, might well be used in combination with other remedies to disinfect the deeper layers of the skin, after the hairs have been thoroughly purged by Dr. Harrison's methods. We would suggest that that plan is thoroughly efficacious, as to the germs in the hairs themselves, but the real difficulty under every method is to reach those which ramify in the lower layers of the epidermis in a more or less albuminoid medium.

\section{ASYLUMS IN INDIA.}

\section{IV.-THE CENTRAL PROVINCES.}

Calcolated at 54 superficial feet per patient, the capacity of the Nagpur and Jubbulpore Asylums is for 162 and 163, and the maximum number of patients in confinement on any one night during 1891 was 135 and 139 respectively, showing that there was no overcrowding at either institution. The movement of population was as follows :-

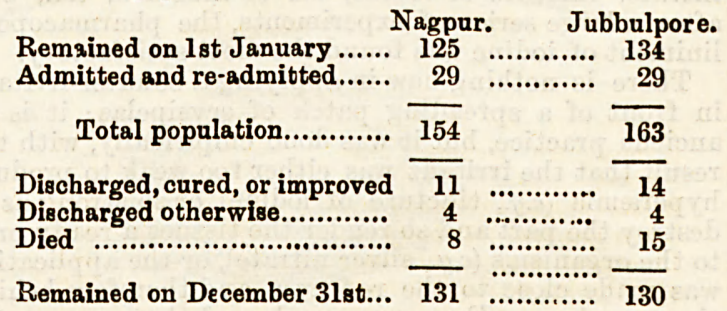

In both asylums the daily average strength was higher than in the previous year, being 127.21 against 118.27 at Nagpur, and 1133.77 against 130.79 at Jubbulpore. The percentage of cured to daily average strength fell at Nagpur from 676 to $3 \cdot 93$, and rose at Jubbulpore from $5 \cdot 35$ to $8 \cdot 97$. Taking the two asylums together this gives a percentage of 6.5 cured to the daily average strength of insane in the Central Provinces. The death-rate at Nagpur, calculated on the daily average strength, fell from $6 \cdot 76$ to $6 \cdot 29$, and at Jub. bulpore rose from 9.94 to $11 \cdot 21$. The total number of criminal insane at Nagpur was 55, with a daily average strength of $46 \cdot 15$, and at Jubbulpore 38 , with a daily average of $31 \cdot 56$.

The financial statements show the total expenditure of the Nagpur Asylum to have been Rs,12,003 13a. 10p., and upon the Jubbulpore Asylum Ks.10,866 9a. 10p., showing the average actual cost per patient as Rs.71 149. 2p. at Nagpur, and Rs.71 0a. 6p. at Jubbulpore. The statistics in general present no specially interesting features.

\section{INSECTS AND GROWING FRUIT.}

INSECTs play a more important part in fruit growing than cultivators generally are aware of. A Washington authority on this subject calls our attention to the necessity of cross fertilization by means of insects before the fruit will set. Pears, for instance, are amongst those fruits which for any prolific bearing require primarily to receive pollen from other varieties through the medium of the passing insect, which, in its search for honey, leaves behind it the necessary pollen, in this manner acting the part of an unconscious, but useful factor in the production of fruit. 